scatter of factories over the countryside, the defence of a group of factories may be an easier problem than the defence of widely scattered units. Merseyside has claims to correct deficiencies in the provision of greater opportunities for skilled male employment and for a greater number of the new 'assembly' industries. For correct industrial balance, Merseyside needs more opportunities for skilled craftsmen, and there are many industries of this type possessing considerable mobility which might be directed to Merseyside if the central government contemplates control of industrial location. Such industries should be directed to the extra-dock zone, where the working conditions ari better, where the product of a foodstuff can be made under more hygienic conditions and where labour lives within easier access of the factory. Moreorer, as regards the industrial satellite in the Mersey. side area, if it is not to drain life from its parent, its success appears to depend on the attraction of new industries from outside Merseyside altogether.

There can be no two opinions as to the value of studies which bring out as clearly for other districts as this one does for Merseyside the factors which determine the volume of employment and local prosperity and well-being, and in their absence it can scarcely be maintained that local authorities are in possession of the knowledge which should be the foundation of their planning. Mr. Smith's report provides an admirable example of the way in which scientific method and investigation can contribute to the solution of major problems in a democratic society.

\section{AUTOMATIC PRODUCTION OF OSCILLATOR SCALES}

$\mathrm{T}$

HE frequency of an oscillator is usually set by dial adjustments corresponding to a calibration chart from which the exact frequency corresponding to the dial indications may be obtained. 'The relationships between the frequency and dial markings, moreover, are different for every oscillator, so that a calibration chart must be prepared for each. This is not particularly difficult but is laborious, by reason of the need to refer for every reading to a calibration chart. It is much simpler to employ a dial marked directly in frequencies, but to provide such a scale by usual methods requires much time and labour when the frequency range is large and the accuracy is to be high. Although the scales for different oscillators are similar, they are not suificiently alike to permit the scales for all oscillators to be printed from a single master film.

A method of printing such scales has, however, been recently developed and has been described by 'T. Slonezewski (Bell Lab. Rec., 20, No. 11, July, 1942). A master scale $A$ is used which, when placed over an unexposed film $B$, is moved with respect to $B$ at a varying rate, such that at each 50 -cycle point a line on scale $A$ will be directly over its correct position on scale $B$. The section of film $B$ is exposed at the instant it is under the corresponding line on film $A$. 'Two oscillators are used, set up facing each other, one serving as the standard and having an accurately marked film scale. The other, to be calibrated, carries on its sprocket the unexposed film that is to be marked, line by line, from the film of the standard, although the spacing of the lines will be different from the standard.

The condenser-driving spindle of each oscillator carries a worm-driven driving gear and film sprocket. For the duration of the calibration, 60-cycle synchronous motors are attached to the condenser worms of each oscillator. A standard motion-picture lamp is mounted behind the film of the standard, and a condensing lens focuses its rays in a narrow band over the section opposite the hair lines where the scale is read. The test oscillator motor receives its supply from the commercial 60-cycle supply. That on the standard is driven by a 'difference' frequency from the output of a modulator to which the output of the two oscillators is fed. When the two oscillators are 60 cycles apart, this latter motor will therefore have a 60-cycle supply, and the two films will be running at the same speed. As each line on the standard film passes through the band of light, it will be printed on the film of the test oscillator. The purpose of this drive arrangement is to vary the speed of the standard film in such a way that the line in the light beam corresponds to the frequency of the test oscillator except for the 60-cycle difference. This 60 -cycle constant difference is readily corrected after calibration by the low-frequency adjustment that has been provided for this purpose.

\section{EARTHED-PLATE AMPLIFIER FOR THE FREQUENCY-MODULATION TRANSMITTER}

IN the United States, frequency-modulation has 1 been assigned the wave band $42-50$ m.c and at these frequencies, simplification in design and reduction in size of transmitters are of particular importance because of lead reactances and stray capacitances to earth, which increase with frequency and with size, and may become serious obstacles in large transmitters operating at high frequencies.

A $10-\mathrm{kw}$. transmitter has been developed recently by the Bell Laboratories in which the difficulties have been overcome using the new Western Electric $389 A A$, air-cooled valve, and by a novel circuit arrangement due to W. H. Doherty. Even with this new tube, however, it was found that there would be sufficient stray plate-earth capacitance to result in a rather large loss in the plate-tuning coil, andof even greater importance-the tuning would be undesirably sharp.

Both these obstacles were overcome by the use of an earthed-plate amplifier and a number of novel features described by A. A. Skene (Bell Lab. Rec., 20, No. 11, July, 1942). The circuit employed results in the stray plate capacitance-to-earth being in parallel with the plate-earthing condenser, which is used to provide a radio-frequency path to earth while blocking the D.C., and thus has no effect on the operation of the plate circuit. Only the filamentto-earth capacitance remains and is very much smaller than the plate-to-earth capacitance. The filament is at a high radio-frequency potential, and it is very necessary to supply filament current without operating the filament transformer at this high potential above earth. It is also necessary to supply radio-frequency driving potential between the grid and filament from the driver unit, one side of which is earthed.

An ingenious arrangement is described which achieves these objectives. The normal plate-tuning coil is replaced in the new circuit by a coil between filament and earth, and is formed of a pair of copper 